\title{
The de la Vallée Poussin Mean and Polynomial Approximation for Exponential Weight
}

\author{
K. Itoh, R. Sakai, and N. Suzuki \\ Department of Mathematics, Meijo University, Tenpaku-ku, Nagoya, Aichi 468-8502, Japan \\ Correspondence should be addressed to K. Itoh; 133451501@ccalumni.meijo-u.ac.jp \\ Received 19 August 2014; Accepted 5 March 2015 \\ Academic Editor: Malte Braack
}

Copyright (C) $2015 \mathrm{~K}$. Itoh et al. This is an open access article distributed under the Creative Commons Attribution License, which permits unrestricted use, distribution, and reproduction in any medium, provided the original work is properly cited.

We study $L^{p}$ boundedness of the de la Vallée Poussin means $v_{n}(f)$ for exponential weight $w(x)=\exp (-Q(x))$ on $\mathbb{R}$. Our main result is $\left\|v_{n}(f)\left(w / T^{1 / 4}\right)\right\|_{L^{p}(\mathbb{R})} \leq C\|f w\|_{L^{p}(\mathbb{R})}$, for every $n \in \mathbb{N}$ and every $1 \leq p \leq \infty$, where $T(x)=x Q^{\prime}(x) / Q(x)$. As an application, we obtain $\lim _{n \rightarrow \infty}\left\|\left(f-v_{n}(f)\right) w / T^{1 / 4}\right\|_{L^{p}(\mathbb{R})}=0$ for $f w \in L^{p}(\mathbb{R})$.

\section{Introduction}

Let $\mathbb{R}=(-\infty, \infty)$ and $\mathbb{N}=\{1,2, \ldots\}$. We consider an exponential weight

$$
w(x)=\exp (-Q(x))
$$

on $\mathbb{R}$, where $Q$ is an even and nonnegative function on $\mathbb{R}$. Throughout this paper we always assume that $w$ belongs to a relevant class $\mathscr{F}\left(C^{2}+\right)$ (see Section 2). We consider a function $T$ defined by

$$
T(x):=\frac{x Q^{\prime}(x)}{Q(x)}, \quad x \neq 0 .
$$

If $T$ is bounded, then $w$ is called a Freud-type weight, and otherwise, $w$ is called an Erdős-type weight.

Let $w=\exp (-Q) \in \mathscr{F}\left(C^{2}+\right)$. By definition, $Q^{\prime \prime}>0$, so that $F(x):=\int_{0}^{1}\left(x u Q^{\prime}(x u) /\left(1-u^{2}\right)^{1 / 2}\right) d u$ is an increasing and continuous function on $[0, \infty)$ with $F(0)=0$ and $\lim _{x \rightarrow \infty} F(x)=\infty$. By the intermediate value theorem, there exists a unique $a_{n}>0$ such that $n=(2 / \pi) F\left(a_{n}\right)$ for every $n \in \mathbb{N}$; that is,

$$
n=\frac{2}{\pi} \int_{0}^{1} \frac{a_{n} u Q^{\prime}\left(a_{n} u\right)}{\left(1-u^{2}\right)^{1 / 2}} d u, \quad n \in \mathbb{N}
$$

holds. This $a_{n}$ is called the Mhaskar-Rakhmanov-Saff number (MRS number). In this sequel, notation $a_{n}$ always stands for the MRS number for a weight $w$ defined by (3) (see [1, page 11], [2, page 180]).

Let $\left\{p_{n}\right\}$ be orthogonal polynomials for a weight $w$; that is, $p_{n}(x)=p_{n}(w, x)$ is the polynomial of degree $n$ such that

$$
\int_{\mathbb{R}} p_{n}(x) p_{m}(x) w^{2}(x) d x=\delta_{m n} .
$$

For $1 \leq p \leq \infty$, we denote by $L^{p}(\mathbb{R})$ the usual $L^{p}$ space on $\mathbb{R}$. For a function $f$ with $f w \in L^{p}(\mathbb{R})$, we set

$$
\begin{aligned}
s_{n}(f)(x) & :=\sum_{k=0}^{n-1} c_{k}(f) p_{k}(x), \text { where } \\
c_{k}(f) & :=\int_{\mathbb{R}} f(t) p_{k}(t) w^{2}(t) d t
\end{aligned}
$$

for $n \in \mathbb{N}$ (the partial sum of Fourier series). The de la Vallée Poussin mean $v_{n}(f)$ of $f$ is defined by

$$
v_{n}(f)(x):=\frac{1}{n} \sum_{j=n+1}^{2 n} s_{j}(f)(x) .
$$

The main result of this paper is the following theorem.

Theorem 1. Let $1 \leq p \leq \infty$. We assume that $w \in \mathscr{F}\left(C^{2}+\right)$ satisfies

$$
T\left(a_{n}\right) \leq c\left(\frac{n}{a_{n}}\right)^{2 / 3}
$$


for some $c>0$. Then there exists a constant $C=C(w, p)>0$ such that when $T^{1 / 4} f w \in L^{p}(\mathbb{R})$, then

$$
\left\|v_{n}(f) w\right\|_{L^{p}(\mathbb{R})} \leq C\left\|T^{1 / 4} f w\right\|_{L^{p}(\mathbb{R})},
$$

and when $f w \in L^{p}(\mathbb{R})$, then

$$
\left\|v_{n}(f) \frac{w}{T^{1 / 4}}\right\|_{L^{p}(\mathbb{R})} \leq C\|f w\|_{L^{p}(\mathbb{R})} .
$$

For Freud-type weights, the following inequality is known:

$$
\left\|v_{n}(f) w\right\|_{L^{p}(\mathbb{R})} \leq C\|f w\|_{L^{p}(\mathbb{R})}
$$

(cf. [3, Lemma 6], [2, Theorem 3.4.2]). Note that when $T$ is bounded, then (7) holds evidently, so (10) follows from (8) or (9) immediately. As for Erdős-type weights, Lubinsky and Mthembu [4] proved (8) for $p=1$, (9) for $p=\infty$, and $\left\|v_{n}(f) w T^{-1 / 4}\right\|_{L^{p}(\mathbb{R})} \leq C\left\|T^{1 / 4} f w\right\|_{L^{p}(\mathbb{R})}$ for $1<p<\infty$ under the assumption $T(x)=O\left(Q^{\prime}(x)^{\varepsilon}\right)$ for every $\varepsilon>0$. (They discussed on Cesaro means $(=(C, 1)$ means $)$ mainly, but it easily ensures the result on de la Vallée Poussin means.) We note that their assumption implies our condition (7) (see Remark 16 in Section 7). Hence our results (8) and (9) improve their result. For more general weights, see [5-7].

Using (9), we have the following result. There exists a constant $C=C(w, p)>0$ such that, for any $w f \in L^{p}(\mathbb{R})$,

$$
\left\|\left(f-v_{n}(f)\right) \frac{w}{T^{1 / 4}}\right\|_{L^{p}(\mathbb{R})} \leq C \inf _{P \in \mathscr{P}_{n}}\|w(f-P)\|_{L^{p}(\mathbb{R})},
$$

where $\mathscr{P}_{n}$ is the set of all polynomials of degree at most $n$. It is known that the right-hand side of (11) attains some $P_{n} \in$ $\mathscr{P}_{n}$; however, it is not easy to determine such $P_{n}$ explicitly. Equation (11) means that the de la Vallée Poussin mean takes the place of $P_{n}$ in some sense; that is, $v_{n}(f)$ is a good concrete approximation polynomial for given function $f$.

This paper is organized as follows. The definition of class $\mathscr{F}\left(C^{2}+\right)$ is given in Section 2. Recalling some estimates for exponential weights in Section 3, we will give a proof of Theorem 1 for the case of $p=\infty$ in Section 4. Basic method of our proof is classic and well known ([3], see also $[4,6])$, but we repeat it in order to make a role of $T(x)$ clear. The complete proof of Theorem 1 is given in Section 5. The estimate (11) is shown in Section 6 together with another application. We discuss the condition (7) in Section 7.

Throughout this paper $C$ will denote a positive constant whose value is not necessarily the same at each occurrence; it may vary even within a line. When we write $C=C(a, b, \ldots)$, then $C$ is a constant which depends on $a, b, \ldots$ only.

\section{Definitions and Notation}

We say that an exponential weight $w=\exp (-Q)$ belongs to class $\mathscr{F}\left(C^{2}+\right)$, when $Q: \mathbb{R} \rightarrow[0, \infty)$ is a continuous and even function and satisfies the following conditions.

(a) $Q^{\prime}(x)$ is continuous in $\mathbb{R}$ with $Q(0)=0$. (b) $Q^{\prime \prime}(x)$ exists and is positive in $\mathbb{R} \backslash\{0\}$.

(c) $\lim _{x \rightarrow \infty} Q(x)=\infty$.

(d) The function $T$ defined in (2) is quasi-increasing in $(0, \infty)$ (i.e., there exists $C>0$ such that $T(x) \leq C T(y)$ whenever $0<x<y$ ), and there exists $\Lambda \in \mathbb{R}$ such that

$$
T(x) \geq \Lambda>1, \quad x \in \mathbb{R} \backslash\{0\} .
$$

(e) There exists $C>0$ such that

$$
\frac{Q^{\prime \prime}(x)}{\left|Q^{\prime}(x)\right|} \leq C \frac{\left|Q^{\prime}(x)\right|}{Q(x)}, \quad \text { a.e. } x \in \mathbb{R}
$$

and there also exist a compact subinterval $J(\ni 0)$ of $\mathbb{R}$ and $C>0$ such that

$$
\frac{Q^{\prime \prime}(x)}{\left|Q^{\prime}(x)\right|} \geq C \frac{\left|Q^{\prime}(x)\right|}{Q(x)}, \quad \text { a.e. } x \in \mathbb{R} \backslash J .
$$

Let $w(x)=\exp (-Q(x)) \in \mathscr{F}\left(C^{2}+\right)$. Suppose that $Q \in$ $C^{3}(\mathbb{R})$ and $\lambda>0$. If there exist $C>0$ and $K>0$ such that for $|x| \geq K$,

$$
\left|\frac{Q^{\prime \prime \prime}(x)}{Q^{\prime \prime}(x)}\right| \leq C\left|\frac{Q^{\prime \prime}(x)}{Q^{\prime}(x)}\right|, \quad \frac{\mid Q^{\prime}(x)}{Q^{\lambda}(x)} \leq C,
$$

then we write $w \in \mathscr{F}_{\lambda}\left(C^{3}+\right)$. Note that if $w$ is a Freud-type, the last inequality holds with $\lambda=1$.

A typical example of Freud-type weight is $w(x)=$ $\exp \left(-|x|^{\alpha}\right)$ with $\alpha>1$. Note that the Hermite polynomials are the orthogonal polynomials for the weight $\exp \left(-|x|^{2}\right)$. Let $u \geq 0, \alpha>0, \alpha+u>1$, and $\ell \in \mathbb{N}$, and we set

$$
Q(x):=|x|^{u}\left(\exp _{\ell}\left(|x|^{\alpha}\right)-\exp _{\ell}(0)\right)
$$

where $\exp _{\ell}(x)=\exp (\exp (\exp (\cdots(\exp x))))(\ell$-times $)$. Then $w(x)=\exp (-Q(x))$ is an Erdös-type weight, which belongs to $\mathscr{F}_{\lambda}\left(C^{3}+\right)$ with $\lambda>1$ (see [8]).

We recall some notation which we use later (cf. [9]). By definition, the partial sum of Fourier series is given by

$$
s_{m}(f)(x)=\int_{\mathbb{R}} K_{m}(x, t) f(t) w^{2}(t) d t,
$$

where

$$
K_{m}(x, t)=\sum_{k=0}^{m-1} p_{k}(x) p_{k}(t)
$$

It is known that, by the Christoffel-Darboux formula,

$$
K_{m}(x, t)=\frac{\gamma_{m-1}}{\gamma_{m}}\left(\frac{p_{m}(x) p_{m-1}(t)-p_{m}(t) p_{m-1}(x)}{x-t}\right),
$$

where $\gamma_{n}$ is the leading coefficient of $p_{n}$; that is, $p_{n}(x)=\gamma_{n} x^{n}+$ $\cdots$. 
The Christoffel function $\lambda_{m}(x)=\lambda_{m}(w, x)$ is defined by

$$
\lambda_{m}(x):=\frac{1}{K_{m}(x, x)}=\left(\sum_{k=0}^{m-1} p_{k}^{2}(x)\right)^{-1}
$$

and $L^{p}$ type Christoffel function $\lambda_{m, p}(x)=\lambda_{m, p}(w, x)$ is defined by

$$
\lambda_{m, p}(x):=\inf _{P \in \mathscr{P}_{m}} \frac{1}{|P(x)|^{p}} \int_{\mathbb{R}}|P(t) w(t)|^{p} d t .
$$

If $p=2$, we know

$$
\lambda_{m, 2}(x)=\lambda_{m}(x)
$$

The MRS numbers $\left\{a_{n}\right\}$ for weight $w$ are monotonically increasing, and we see easily

$$
\lim _{n \rightarrow \infty} a_{n}=\infty, \quad \lim _{n \rightarrow \infty} \frac{a_{n}}{n}=0 .
$$

Moreover, for $n \in \mathbb{N}$, there exists $C>0$ independent of $n$, such that

$$
\frac{a_{n}}{C} \leq \frac{\gamma_{n-1}}{\gamma_{n}} \leq C a_{n}
$$

(see [9, Lemma 13.9]). We also use the following functions:

$$
\Phi_{n}(x):= \begin{cases}\frac{1-|x| / a_{2 n}}{\sqrt{1-|x| / a_{n}+\delta_{n}}}, & |x| \leq a_{n}, \\ \Phi_{n}\left(a_{n}\right), & a_{n}<|x|,\end{cases}
$$

where $\delta_{n}:=\left\{n T\left(a_{n}\right)\right\}^{-2 / 3}$ and

$$
\varphi_{n}(x):=\frac{a_{n}}{n} \Phi_{n}(x) .
$$

\section{Lemmas}

We recall some basic estimates.

Lemma 2 (infinite-finite range inequality [9, Theorem 1.9(a)]). Let $w \in \mathscr{F}\left(C^{2}+\right), 0<p \leq \infty$ and $P \in \mathscr{P}_{n}(n \geq 1)$. Then

$$
\|P w\|_{L^{p}(\mathbb{R})} \leq 2\|P w\|_{L^{p}\left(|x|<a_{n}\right)} .
$$

Lemma 3 (see [10, Lemma 3.4]). Let $w \in \mathscr{F}\left(C^{2}+\right)$. There exists a constant $C=C(w)>0$ such that

$$
\frac{a_{n}}{n} \frac{1}{\sqrt{T(x)} \varphi_{n}(x)} \leq C,
$$

and hence

$$
\frac{1}{\sqrt{T(x)}} \leq C \Phi_{n}(x)
$$

holds for every $n \in \mathbb{N}$ and $x>0$.
Lemma 4 (see [9, Theorem 9.3]). Let $w \in \mathscr{F}\left(C^{2}+\right)$, and $0<$ $p \leq \infty$. Then there exists a constant $C=C(w, p)>0$ such that, for every $n \in \mathbb{N}$ and $x \in \mathbb{R}$,

$$
\lambda_{n, p}(x) \geq C \varphi_{n}(x) w^{p}(x)
$$

and when $|x| \leq a_{n}$, one has

$$
\frac{1}{C} \varphi_{n}(x) w^{p}(x) \leq \lambda_{n, p}(x) \leq C \varphi_{n}(x) w^{p}(x) .
$$

Lemma 5. Let $w \in \mathscr{F}\left(C^{2}+\right)$. Then there exist $0<c_{0}<1$ and $C=C(w)>0$ such that

$$
\frac{1}{C} T(x) \leq T\left(x \pm \frac{c_{0}}{T(x)}\right) \leq C T(x)
$$

for every $x \in \mathbb{R}$.

Proof. It is known that there exist $0<c_{0}<1$ and $C=C(w)>$ 0 such that

$$
\frac{1}{C} T(x) \leq T\left(x\left[1 \pm \frac{c_{0}}{T(x)}\right]\right) \leq C T(x)
$$

for every $x \in \mathbb{R}[9$, Theorem 3.2(e)]. If $|x| \leq 1$, then

$$
\left|x \pm \frac{c_{0}}{T(x)}\right| \leq|x|+c_{0} \leq 2,
$$

and hence (32) holds evidently. If $|x|>1$, then

$$
|x|\left(1+\frac{c_{0}}{T(x)}\right) \geq\left|x \pm \frac{c_{0}}{T(x)}\right| \geq|x|\left(1-\frac{c_{0}}{T(x)}\right) .
$$

Since $T$ is quasi-increasing, (33) shows (32).

Lemma 6 (see [10, Theorem 3.2]). If $w \in \mathscr{F}\left(C^{2}+\right)$ is an Erdöstype weight, then for any $\eta>0$ there exists a constant $C=$ $C(w, \eta)>0$ such that

$$
a_{n} \leq \mathrm{Cn} n^{\eta}
$$

for every $n \in \mathbb{N}$.

Lemma 7 (see [9, Lemma 3.5(a), (b) and Lemma 3.11(3.52)]). Let $w \in \mathscr{F}\left(C^{2}+\right)$. There exists a constant $C=C(w)>0$ such that

$$
\begin{aligned}
a_{2 n} & \leq C a_{n}, \\
T\left(a_{2 n}\right) & \leq C T\left(a_{n}\right), \\
\frac{1}{C} \frac{a_{n}}{T\left(a_{n}\right)} & \leq a_{2 n}-a_{n}
\end{aligned}
$$

for every $n \in \mathbb{N}$.

\section{Proof of Theorem 1 for $p=\infty$}

We begin with the following proposition. 
Proposition 8. Let $w \in \mathscr{F}\left(C^{2}+\right)$. Then there exists a constant $C=C(w)>0$ such that

$$
\frac{w^{2}(x)}{\sqrt{T(x)}} \sum_{k=0}^{n-1} p_{k}^{2}(x) \leq C \frac{n}{a_{n}}
$$

for every $x \in \mathbb{R}$ and every $n \in \mathbb{N}$.

Proof. From Lemmas 3 and 4 for $p=2$, the proposition follows immediately.

The following estimate is known essentially (see [6, Theorem 4], [7, Theorem 1.2] and [5, Lemma 1]), but we give a proof for the sake of completeness.

Theorem 9. Let $w \in \mathscr{F}\left(C^{2}+\right)$. Then there exists a constant $C=C(w)>0$ such that

$$
\left\|v_{n}(f) w \Phi_{2 n}^{1 / 2}\right\|_{L^{\infty}(\mathbb{R})} \leq C\|f w\|_{L^{\infty}(\mathbb{R})}
$$

for any $w f \in L^{\infty}(\mathbb{R})$ and $n \in \mathbb{N}$.

Proof. Let $x \in \mathbb{R}$ and $n, m \in \mathbb{N}$ with $n-1 \leq m \leq 2 n$. We set

$$
\begin{gathered}
g(t):=f(t) \chi_{\left(x-a_{n} / n, x+a_{n} / n\right)}(t), \\
h(t):=f(t)-g(t),
\end{gathered}
$$

where $\chi_{(a, b)}$ is the characteristic function of a set $(a, b)$. Then

$$
v_{n}(f)(x)=v_{n}(g)(x)+v_{n}(h)(x) .
$$

We first consider an estimate of $v_{n}(g)$. By the Schwarz inequality

$$
\begin{aligned}
& \left|s_{m}(g)(x)\right| \\
& \quad=\left|\int_{x-a_{n} / n}^{x+a_{n} / n} f(t) K_{m}(x, t) w^{2}(t) d t\right| \\
& \quad \leq\|f w\|_{L^{\infty}(\mathbb{R})} \int_{x-a_{n} / n}^{x+a_{n} / n}\left|K_{m}(x, t)\right| w(t) d t \\
& \quad \leq\|f w\|_{L^{\infty}(\mathbb{R})}\left(\frac{2 a_{n}}{n}\right)^{1 / 2}\left(\int_{\mathbb{R}} K_{m}^{2}(x, t) w^{2}(t) d t\right)^{1 / 2},
\end{aligned}
$$

and since

$$
\int_{\mathbb{R}} K_{m}^{2}(x, t) w^{2}(t) d t=\sum_{i=0}^{m-1} p_{i}^{2}(x) \leq \sum_{i=0}^{2 n-1} p_{i}^{2}(x)=\frac{1}{\lambda_{2 n}(x)},
$$

(26) and (30) give us

$$
\begin{aligned}
\left|w(x) s_{m}(g)(x) \Phi_{2 n}^{1 / 2}(x)\right| & \leq\|f w\|_{L^{\infty}(\mathbb{R})}\left(\frac{2 \varphi_{2 n}(x)}{\lambda_{2 n}(x)} w^{2}(x)\right)^{1 / 2} \\
& \leq C\|f w\|_{L^{\infty}(\mathbb{R})},
\end{aligned}
$$

so that

$$
\left|v_{n}(g)(x) w(x) \Phi_{2 n}^{1 / 2}(x)\right| \leq C\|f w\|_{L^{\infty}(\mathbb{R})} .
$$

Next, for an estimate of $v_{n}(h)(x)$, we set

$$
H(t):=\frac{h(t)}{x-t}
$$

and denote by $\left\{b_{j}(H)\right\}$ the Fourier coefficients of $H$; that is,

$$
b_{j}(H):=\int_{\mathbb{R}} H(t) p_{j}(t) w^{2}(t) d t .
$$

Using the Christoffel-Darboux formula (19), we have

$$
\begin{aligned}
& \left|s_{m}(h)(x)\right| \\
& =\mid \int_{\mathbb{R}} \frac{\gamma_{m-1}}{\gamma_{m}}\left(\frac{p_{m}(x) p_{m-1}(t)-p_{m}(t) p_{m-1}(x)}{x-t}\right) \\
& \cdot h(t) w^{2}(t) d t \mid \\
& =\frac{\gamma_{m-1}}{\gamma_{m}} \mid p_{m}(x) \int_{\mathbb{R}} p_{m-1}(t) H(t) w^{2}(t) d t-p_{m-1}(x) \\
& \leq \frac{\gamma_{m-1}}{\gamma_{m}}\left(\left|p_{m}(x)\right|\left|b_{m-1}(H)\right|+\left|p_{m-1}(x)\right|\left|b_{m}(H)\right|\right) .
\end{aligned}
$$

By (24) and the Schwarz inequality, we have

$$
\begin{aligned}
\left|v_{n}(h)(x)\right| & \leq \frac{1}{n} \max _{m \leq 2 n}\left\{\frac{\gamma_{m-1}}{\gamma_{m}}\right\} \times \frac{2}{\lambda_{2 n}^{1 / 2}(x)}\left(\sum_{m=0}^{2 n} b_{m}^{2}(H)\right)^{1 / 2} \\
& \leq C \frac{a_{n}}{n} \frac{1}{\lambda_{2 n}^{1 / 2}(x)}\left(\sum_{m=0}^{2 n} b_{m}^{2}(H)\right)^{1 / 2} .
\end{aligned}
$$

Since

$$
\begin{aligned}
\sum_{m=0}^{2 n} b_{m}^{2}(H) & \leq \sum_{m=0}^{\infty} b_{m}^{2}(H) \\
& \leq \int_{\mathbb{R}}(H(t) w(t))^{2} d t \\
& =\int_{|t-x| \geq a_{n} / n}\left(\frac{f(t) w(t)}{t-x}\right)^{2} d t \\
& \leq\|f w\|_{L^{\infty}(\mathbb{R})}^{2} \frac{2 n}{a_{n}},
\end{aligned}
$$


(26) and (30) imply

$$
\begin{aligned}
\left|v_{n}(h)(x) w(x)\right| & \leq C \frac{a_{n}}{n}\left(\frac{w^{2}(x)}{\lambda_{2 n}(x)}\right)^{1 / 2}\|f w\|_{L^{\infty}(\mathbb{R})} \sqrt{\frac{n}{a_{n}}} \\
& \leq C\|f w\|_{L^{\infty}(\mathbb{R})} \frac{1}{\Phi_{2 n}^{1 / 2}(x)}\left(\frac{w^{2}(x) \varphi_{2 n}(x)}{\lambda_{2 n}(x)}\right)^{1 / 2} \\
& \leq C\|f w\|_{L^{\infty}(\mathbb{R})} \frac{1}{\Phi_{2 n}^{1 / 2}(x)} .
\end{aligned}
$$

This together with (47) gives us

$$
\left|v_{n}(f)(x) w(x) \Phi_{2 n}^{1 / 2}(x)\right| \leq C\|f w\|_{L^{\infty}(\mathbb{R})},
$$

which completes the proof of (41).

The following corollary contains (9) for $p=\infty$.

Corollary 10. Let $w \in \mathscr{F}\left(C^{2}+\right)$. Then there exists a constant $C=C(w)>0$ such that

$$
\left\|v_{n}(f) \frac{w}{T^{1 / 4}}\right\|_{L^{\infty}(\mathbb{R})} \leq C\|f w\|_{L^{\infty}(\mathbb{R})}
$$

for any $w f \in L^{\infty}(\mathbb{R})$.

Proof. From Theorem 9 and Lemma 3, this corollary follows immediately.

We give a proof of (8) for $p=\infty$.

Theorem 11. Let $w \in \mathscr{F}\left(C^{2}+\right)$ such that $T\left(a_{n}\right) \leq c\left(n / a_{n}\right)^{2 / 3}$ with some $c>0$. Then there exists a constant $C=C(w)>0$ such that if $T^{1 / 4} w f \in L^{\infty}(\mathbb{R})$, one has

$$
\left\|v_{n}(f) w\right\|_{L^{\infty}(\mathbb{R})} \leq C\left\|T^{1 / 4} f w\right\|_{L^{\infty}(\mathbb{R})} .
$$

Proof. Let $x \in \mathbb{R}$ and $n, m \in \mathbb{N}$ such that $n-1 \leq$ $m \leq 2 n$. As in the proof of Theorem 9, we set $g(t):=$ $f(t) \chi_{\left(x-a_{n} / n, x+a_{n} / n\right)}(t), h(t):=f(t)-g(t)$. Then $v_{n}(f)(x)=$ $v_{n}(g)(x)+v_{n}(h)(x)$. Since $v_{n}(g), v_{n}(h) \in \mathscr{P}_{2 n}$, by Lemma 2, we may suppose that $|x| \leq a_{2 n}$.

We first prove that, for any $n \in \mathbb{N}$,

$$
T(x) \leq C T(t)
$$

whenever $|x| \leq a_{2 n}$ and $|x-t| \leq a_{n} / n$. In fact, we now denote by $C_{1}>0$ a constant in Lemma 7 . Since $T$ is quasi-increasing, there exists a constant $C_{2}>0$ such that $T(x) \leq C_{2} T(y)$ whenever $0 \leq x \leq y$. Then by (7), there exists $N_{0} \in \mathbb{N}$ such that $n \geq C_{1}^{2} T\left(a_{n}\right)$ holds for every $n \geq N_{0}$, so that Lemma 7 gives us

$$
|t| \leq|x|+\frac{a_{n}}{n} \leq a_{2 n}+\frac{1}{C_{1}^{2}} \frac{a_{n}}{T\left(a_{n}\right)} \leq a_{2 n}+\frac{1}{C_{1}} \frac{a_{2 n}}{T\left(a_{2 n}\right)} \leq a_{4 n} .
$$

This together with (38) and (7) implies

$$
T(|t|) \leq C_{2} T\left(a_{4 n}\right) \leq C_{1}^{2} C_{2} T\left(a_{n}\right) \leq c C_{1}^{2} C_{2}\left(\frac{n}{a_{n}}\right)^{2 / 3} .
$$

Since $n / a_{n} \rightarrow \infty$ as $n \rightarrow \infty$, we may assume that

$$
c C_{1}^{2} C_{2}\left(\frac{n}{a_{n}}\right)^{2 / 3} \leq c_{0} \frac{n}{a_{n}}
$$

holds for every $n \geq N_{0}$ if we take $N_{0}$ larger as needed, where $c_{0}$ is the constant in Lemma 5. Then

$$
|x| \leq \frac{a_{n}}{n}+|t| \leq \frac{c_{0}}{T(t)}+|t|,
$$

and hence Lemma 5 gives us

$$
T(x)=T(|x|) \leq C_{2} T\left(\frac{c_{0}}{T(t)}+|t|\right) \leq C_{2} C T(|t|)=C T(t) .
$$

When $n \leq N_{0}$, then $1 \leq T(x) \leq C$ for $|x| \leq a_{2 N_{0}}$, so that (57) holds immediately.

Now we estimate $v_{n}(g)(x)$. By the Schwarz inequality, (57), and Proposition 8,

$$
\begin{aligned}
\left|s_{m}(g)(x) w(x)\right| \leq & C\left(\sum_{k=0}^{m-1} \frac{w^{2}(x)}{T^{1 / 2}(x)} p_{k}^{2}(x)\right)^{1 / 2} \\
& \cdot\left(\int_{x-a_{n} / n}^{x+a_{n} / n}\left|T^{1 / 4}(t) w(t) f(t)\right|^{2} d t\right)^{1 / 2} \\
\leq & C \sqrt{\frac{m}{a_{m}}}\left\|T^{1 / 4} w f\right\|_{L^{\infty}(\mathbb{R})}\left(\int_{x-a_{n} / n}^{x+a_{n} / n} d t\right)^{1 / 2} \\
\leq & C\left\|T^{1 / 4} w f\right\|_{L^{\infty}(\mathbb{R})},
\end{aligned}
$$

which implies

$$
\left|v_{n}(g)(x) w(x)\right| \leq C\left\|T^{1 / 4} f w\right\|_{L^{\infty}(\mathbb{R})} .
$$

Next, we estimate $v_{n}(h)(x)$. Since

$$
\begin{aligned}
v_{n} & (h)(x) \\
= & \frac{1}{n} \sum_{m=n+1}^{2 n} \frac{\gamma_{m-1}}{\gamma_{m}}\left(b_{m-1}(H) p_{m}(x)-b_{m}(H) p_{m-1}(x)\right)
\end{aligned}
$$

as before, where $H(t)=h(t) /(x-t)$, Proposition 8 gives us

$$
\begin{aligned}
& \left|w(x) v_{n}(h)(x)\right| \\
& \leq \frac{2 a_{n}}{n}\left(\sum_{m=0}^{2 n} w^{2}(x) \sum_{m=n}^{2 n} p_{m}^{2}(x)\right)^{1 / 2}\left(\sum_{m=0}^{2 n} b_{m}^{2}(H)\right)^{1 / 2} \\
& \quad \leq C \sqrt{\frac{a_{n}}{n}}\left(T^{1 / 2}(x) \int_{|x-t|>a_{n} / n}\left|\frac{f(t) w(t)}{x-t}\right|^{2} d t\right)^{1 / 2} .
\end{aligned}
$$


By Lemma 5, we can take $c>0$ small enough such that $T(x) \geq$ $C T(x \pm c / T(x))$ for $C>0$. Then we have

$$
\begin{aligned}
& T^{1 / 2}(x) \int_{c / T(x)>|x-t|>a_{n} / n} \frac{|f(t) w(t)|^{2}}{(x-t)^{2}} d t \\
& \leq C T^{1 / 2}(x) \int_{c / T(x)>|x-t|>a_{n} / n} \frac{1}{T^{1 / 2}(|x-c / T(x)|)} \\
& \leq C\left\|T^{1 / 4} f w\right\|_{L^{\infty}(\mathbb{R})}^{2} \int_{c / T(x)>|x-t|>a_{n} / n} \cdot \frac{\left|f(t) T^{1 / 4}(t) w(t)\right|^{2}}{(x-t)^{2}} d t \\
& \leq C \frac{n}{a_{n}}\left\|T^{1 / 4} f w\right\|_{L^{\infty}(\mathbb{R})}^{2}
\end{aligned}
$$

Also since $T\left(a_{n}\right) \leq C\left(n / a_{n}\right)^{2 / 3}$ and $|x| \leq a_{2 n}$, we have

$$
\begin{aligned}
& T^{1 / 2}(x) \int_{\mathcal{C} / T(x) \leq|x-t|} \frac{|f(t) w(t)|^{2}}{(x-t)^{2}} d t \\
& \quad \leq\|f w\|_{L^{\infty}(\mathbb{R})}^{2} T^{1 / 2}(x) \int_{c / T(x) \leq|x-t|} \frac{1}{(x-t)^{2}} d t \\
& \quad \leq C\|f w\|_{L^{\infty}(\mathbb{R})}^{2} T^{3 / 2}(x) \leq C \frac{n}{a_{n}}\|f w\|_{L^{\infty}(\mathbb{R})}^{2} .
\end{aligned}
$$

These estimates show

$$
\begin{gathered}
\left(T^{1 / 2}(x) \int_{|x-t|>a_{n} / n}\left|\frac{f(t) w(t)}{x-t}\right|^{2} d t\right)^{1 / 2} \\
\leq C \sqrt{\frac{n}{a_{n}}}\left\|T^{1 / 4} f w\right\|_{L^{\infty}(\mathbb{R})},
\end{gathered}
$$

and hence

$$
\left\|v_{n}(h) w\right\|_{L^{\infty}(\mathbb{R})} \leq C\left\|T^{1 / 4} f w\right\|_{L^{\infty}(\mathbb{R})}
$$

follows. This together with (64) implies (56).

\section{Proof of Theorem 1 for $1 \leq p<\infty$}

In this section we complete the proof of Theorem 1.

Proof of (8). The $L^{\infty}$-norm case is Theorem 11 . We prove the $L^{1}$-norm case. By the duality of $L^{1}$-norm,

$$
\left\|v_{n}(f) w\right\|_{L^{1}(\mathbb{R})}=\sup _{\|g w\|_{L^{\infty}(\mathbb{R})} \leq 1} \int_{\mathbb{R}} v_{n}(f)(x) g(x) w(x)^{2} d x .
$$

Since $K_{m}(x, t)=K_{m}(t, x)$, we see

$$
\begin{aligned}
\int_{\mathbb{R}} & s_{m}(f)(x) g(x) w^{2}(x) d x \\
= & \int_{\mathbb{R}} f(x) s_{m}(g)(x) w^{2}(x) d x,
\end{aligned}
$$

and hence

$$
\int_{\mathbb{R}} v_{n}(f)(x) g(x) w^{2}(x) d x=\int_{\mathbb{R}} f(x) v_{n}(g)(x) w^{2}(x) d x .
$$

Therefore, using Corollary 10, we have

$$
\begin{aligned}
& \left\|v_{n}(f) w\right\|_{L^{1}(\mathbb{R})} \\
& =\sup _{\|g w\|_{L^{\infty}(\mathbb{R})} \leq 1} \int_{\mathbb{R}} v_{n}(f)(x) g(x) w^{2}(x) d x \\
& =\sup _{\|g w\|_{L^{\infty}(\mathbb{R})} \leq 1} \int_{\mathbb{R}} f(x) v_{n}(g)(x) w^{2}(x) d x \\
& \leq \sup _{\|g w\|_{L^{\infty}(\mathbb{R})} \leq 1}\left\|T^{1 / 4} f w\right\|_{L^{1}(\mathbb{R})}\left\|\frac{1}{T^{1 / 4}} v_{n}(g) w\right\|_{L^{\infty}(\mathbb{R})} \\
& \leq \sup _{\|g w\|_{L^{\infty}(\mathbb{R})} \leq 1}\left\|T^{1 / 4} f w\right\|_{L^{1}(\mathbb{R})}\|g w\|_{L^{\infty}(\mathbb{R})} \\
& \leq\left\|T^{1 / 4} f w\right\|_{L^{1}(\mathbb{R})} \cdot
\end{aligned}
$$

Since the operator norms

$$
F \longmapsto w v_{n}\left(F \frac{1}{w T^{1 / 4}}\right)
$$

for $p=1$ and $p=\infty$ are bounded, the Riesz-Thorin interpolation theorem gives us

$$
\sup _{\|F\|_{L^{p}(\mathbb{R})} \leq 1}\left\|w v_{n}\left(F \frac{1}{w T^{1 / 4}}\right)\right\|_{L^{p}(\mathbb{R})}<\infty
$$

for every $p$ with $1 \leq p \leq \infty$. This implies (8).

Proof of (9). The $L^{\infty}$-norm case is Corollary 10 . Now, we show $L^{1}$-norm case. Similar as above,

$$
\begin{aligned}
& \left\|v_{n}(f) \frac{w}{T^{1 / 4}}\right\|_{L^{1}(\mathbb{R})} \\
& =\sup _{\|w g\|_{L^{\infty}(\mathbb{R})} \leq 1} \int_{\mathbb{R}} v_{n}(f)(x) \frac{g(x)}{T(x)^{1 / 4}} w^{2}(x) d x \\
& =\sup _{\|w g\|_{L^{\infty}(\mathbb{R})} \leq 1} \int_{\mathbb{R}} f(x) v_{n}\left(\frac{g}{T^{1 / 4}}\right)(x) w^{2}(x) d x \\
& \leq \sup _{\|w g\|_{L^{\infty}(\mathbb{R})} \leq 1}\left\|w v_{n}\left(\frac{g}{T^{1 / 4}}\right)\right\|_{L^{\infty}(\mathbb{R})}\|f w\|_{L^{1}(\mathbb{R})} .
\end{aligned}
$$

Since

$$
\begin{aligned}
\left\|w v_{n}\left(\frac{g}{T^{1 / 4}}\right)\right\|_{L^{\infty}(\mathbb{R})} & \leq C\left\|T^{1 / 4} \frac{g}{T^{1 / 4}} w\right\|_{L^{\infty}(\mathbb{R})} \\
& =C\|g w\|_{L^{\infty}(\mathbb{R})} \\
& \leq C
\end{aligned}
$$


by (56), we see

$$
\left\|v_{n}(f) \frac{w}{T^{1 / 4}}\right\|_{L^{1}(\mathbb{R})} \leq C\|f w\|_{L^{1}(\mathbb{R})} .
$$

Hence by the Riesz-Thorin interpolation theorem for the operator

$$
F \longmapsto \frac{w}{T^{1 / 4}} v_{n}\left(w^{-1} F\right)
$$

we have

$$
\sup _{\|F\|_{L^{p}(\mathbb{R})} \leq 1}\left\|\frac{w}{T^{1 / 4}} v_{n}\left(w^{-1} F\right)\right\|_{L^{p}(\mathbb{R})}<\infty
$$

for every $1 \leq p \leq \infty$. This implies (9).

\section{Corollaries}

We begin with the following corollary.

Corollary 12. Let $1 \leq p \leq \infty$ and $w \in \mathscr{F}\left(C^{2}+\right)$ satisfy (7). Then there exists a constant $C=C(w, p)>0$ such that, for every $w f \in L^{p}(\mathbb{R})$ and $n \in \mathbb{N}$,

$$
\left\|v_{n}(f) w\right\|_{L^{p}(\mathbb{R})} \leq C T^{1 / 4}\left(a_{n}\right)\|f w\|_{L^{p}(\mathbb{R})} .
$$

Proof. By Lemma 2 and (9) in Theorem 1,

$$
\begin{aligned}
\left\|v_{n}(f) w\right\|_{L^{p}(\mathbb{R})} & \leq C\left\|v_{n}(f) w\right\|_{L^{p}\left(\left[-a_{2 n}, a_{2 n}\right]\right)} \\
& \leq C T^{1 / 4}\left(a_{n}\right)\left\|v_{n}(f) \frac{w}{T^{1 / 4}}\right\|_{L^{p}(\mathbb{R})} \\
& \leq C T^{1 / 4}\left(a_{n}\right)\|f w\|_{L^{p}(\mathbb{R})}
\end{aligned}
$$

holds for every $1 \leq p \leq \infty$.

To discuss polynomial approximations, we define the degree of weighted polynomial approximation for $w f \epsilon$ $L^{p}(\mathbb{R})$ by

$$
E_{p, n}(w, f):=\inf _{P \in \mathscr{P}_{n}}\|w(f-P)\|_{L^{p}(\mathbb{R})} .
$$

We quote two results from our previous papers.

Proposition 13 (see [11, Theorem 1] and [10, Theorem 6.1]). Let $1 \leq p \leq \infty$ and $w \in \mathscr{F}\left(C^{2}+\right)$. Then there exists a constant $C=C(w, p)>0$ such that for every $n \in N$, if $f$ is absolutely continuous and $f^{\prime} w \in L^{p}(\mathbb{R})$, then

$$
E_{p, n}(w, f) \leq C \frac{a_{n}}{n}\left\|f^{\prime} w\right\|_{L^{p}(\mathbb{R})},
$$

and if $P \in \mathscr{P}_{n}$, then

$$
\left\|\frac{1}{\sqrt{T}} P^{\prime} w\right\|_{L^{p}(\mathbb{R})} \leq C \frac{n}{a_{n}}\|P w\|_{L^{p}(\mathbb{R})}
$$

holds true.
Corollary 14. Let $1 \leq p \leq \infty$ and $w \in \mathscr{F}\left(C^{2}+\right)$ satisfy (7). Then there exists a constant $C=C(w, p)>0$ such that, for every $n \in \mathbb{N}$ and every $w f \in L^{p}(\mathbb{R})$,

$$
\begin{array}{r}
\left\|\left(f-v_{n}(f)\right) \frac{w}{T^{1 / 4}}\right\|_{L^{p}(\mathbb{R})} \leq C E_{p, n}(w, f), \\
\left\|\left(f-v_{n}(f)\right) w\right\|_{L^{p}(\mathbb{R})} \leq C T^{1 / 4}\left(a_{n}\right) E_{p, n}(w, f),
\end{array}
$$

and when $T^{1 / 4} w f \in L^{p}(\mathbb{R})$,

$$
\left\|\left(f-v_{n}(f)\right) w\right\|_{L^{p}(\mathbb{R})} \leq C E_{p, n}\left(T^{1 / 4} w, f\right) .
$$

Moreover if $f$ is absolutely continuous and $f^{\prime} w \in L^{p}(\mathbb{R})$, then

$$
\left\|\left(f-v_{n}(f)\right) \frac{w}{T^{1 / 4}}\right\|_{L^{p}(\mathbb{R})} \leq C \frac{a_{n}}{n}\left\|f^{\prime} w\right\|_{L^{p}(\mathbb{R})} .
$$

Proof. Since $v_{n}(P)=P$ for every $P \in \mathscr{P}_{n}$, we have $v_{n}(f)=$ $P+v_{n}(f-P)$, and hence (9) gives us

$$
\begin{aligned}
& \left\|\left(f-v_{n}(f)\right) \frac{w}{T^{1 / 4}}\right\|_{L^{p}(\mathbb{R})} \\
& \leq\left\|(f-P) \frac{w}{T^{1 / 4}}\right\|_{L^{p}(\mathbb{R})}+\left\|v_{n}(f-P) \frac{w}{T^{1 / 4}}\right\|_{L^{p}(\mathbb{R})} \\
& \quad \leq\left\|(f-P) \frac{w}{T^{1 / 4}}\right\|_{L^{p}(\mathbb{R})}+\|(f-P) w\|_{L^{p}(\mathbb{R})} \\
& \quad \leq C\|(f-P) w\|_{L^{p}(\mathbb{R})},
\end{aligned}
$$

which shows (87). By using (82) and (8), we also obtain (88) and (89), respectively. Favard-type inequality (90) follows from (85) and (87).

Combining (8) with (86), we obtain the following estimate of the derivative of $v_{n}(f)$.

Corollary 15. Let $1 \leq p \leq \infty$ and $w \in \mathscr{F}\left(C^{2}+\right)$ satisfy (7). Then there exists a constant $C=C(w, p)>0$ such that for every $n \in \mathbb{N}$

$$
\left\|\frac{1}{\sqrt{T}} v_{n}^{\prime}(f) w\right\|_{L^{p}(\mathbb{R})} \leq C \frac{n}{a_{n}}\left\|T^{1 / 4} f w\right\|_{L^{p}(\mathbb{R})} .
$$

\section{Remarks}

In this final section we make three remarks. First one is concerning the condition (7) in Theorem 1.

Remark 16. Let $w=\exp (-Q) \in \mathscr{F}\left(C^{2}+\right)$.

(1) Let $0<\lambda<2$. If $w$ satisfies

$$
\frac{\left|Q^{\prime}(x)\right|}{Q^{\lambda}(x)} \leq C \quad(|x| \geq 1)
$$

for some $C>0$. Then the condition (7) holds true. In particular, all $w \in \mathscr{F}_{\lambda}\left(C^{3}+\right)$ satisfy (7).

(2) If $T(x)=O\left(\left(Q^{\prime}(x)\right)^{\varepsilon}\right)$ for some $0<\varepsilon<1 / 2$, then (93) holds for some $0<\lambda<2$. In particular, all the weights discussed in [4] satisfy (7). 
In fact, if $w$ is Freud-type, then (7) holds clearly, so that we may assume that $w$ is Erdös-type. In [10, Lemma 3.2], we showed that when $w$ satisfies (93), then $T\left(a_{n}\right) \leq C n^{2 / 3-\delta}$ holds for some $0<\delta<2 / 3$. Hence this and (36) imply (7). Since $\left|x Q^{\prime}(x)\right| / Q(x)=T(x) \leq C\left|Q^{\prime}(x)\right|^{\varepsilon}, \lambda:=1 /(1-\varepsilon)<2$ satisfies (93).

(3) The condition (7) is equivalent to

$$
\left(a_{n} n^{2}\right)^{1 / 3} \leq \mathrm{CQ}\left(a_{n}\right)
$$

with some constant $C>0$, because $Q\left(a_{n}\right) \sim n / \sqrt{T\left(a_{n}\right)}$ (see $[1$, Lemma 3.4(3.18)]).

Next we remark on the degree of weighted polynomial approximation $E_{p, n}(w, f)$.

Remark 17. Let $1 \leq p \leq \infty$. It is known that if $w \in \mathscr{F}\left(C^{2}+\right)$, then $\lim _{n \rightarrow \infty} E_{p, n}(w, f)=0$ for $w f \in L^{p}(\mathbb{R})$ (when $p=\infty$ we further assume that $\lim _{|x| \rightarrow \infty} f(x) w(x)=0$ ) (e.g., [1, Theorem 1.4]). Hence (87) implies

$$
\lim _{n \rightarrow \infty}\left\|\left(f-v_{n}(f)\right) \frac{w}{T^{1 / 4}}\right\|_{L^{p}(\mathbb{R})}=0 .
$$

This is a concrete polynomial approximation for a given function $f$. Similar argument can not apply to (89), because $T^{1 / 4} w$ may not belong to $\mathscr{F}\left(C^{2}+\right)$. To overcome this difficulty, we use a mollification of a weight ([10, Theorem 4.1]): let $w \in \mathscr{F}_{\lambda}\left(C^{3}+\right)$ with $0<\lambda \leq 3 / 2$. Then we can construct a new weight $w^{*} \in \mathscr{F}\left(C^{2}+\right)$ which satisfies $T^{1 / 4} w \sim w^{*}$, $a_{n} \sim a_{n}^{*}$, and $T \sim T^{*}$, where $a_{n}^{*}$ and $T^{*}$ are the MRS number and a function defined (2) with respect to $w^{*}$, respectively. Hence $E_{p, n}\left(T^{1 / 4} w, f\right) \leq C E_{p, n}\left(w^{*}, f\right)$ and (89) show that if $w \in \mathscr{F}_{\lambda}\left(C^{3}+\right)$ with $0<\lambda \leq 3 / 2$ and if $T^{1 / 4} w f \in L^{p}(\mathbb{R})$, then

$$
\lim _{n \rightarrow \infty}\left\|\left(f-v_{n}(f)\right) w\right\|_{L^{p}(\mathbb{R})}=0
$$

(when $p=\infty$ we further assume that $\left.\lim _{|x| \rightarrow \infty} T^{1 / 4}(x) f(x) w(x)=0\right)$.

Remark 18. Equation (92) suggests that the following inequality would be true:

$$
\left\|v_{n}^{\prime}(f) w\right\|_{L^{p}(\mathbb{R})} \leq C \frac{n}{a_{n}}\left\|T^{3 / 4} f w\right\|_{L^{p}(\mathbb{R})} .
$$

We will discuss this estimate elsewhere.

\section{Conflict of Interests}

The authors declare that there is no conflict of interests regarding the publication of this paper.

\section{References}

[1] D. S. Lubinsky, "A survey of weighted polynomial approximation with exponential weights," Surveys in Approximation Theory, vol. 3, pp. 1-105, 2007.
[2] H. N. Mhaskar, Introduction to the Theory of Weighted Polynomial Approximation, World Scientific, 1996.

[3] G. Freud, "On Polynomial approximation with the weight $\exp \left(-x^{2 k} / 2\right)$," Acta Mathematica Academiae Scientiarum Hungaricae, vol. 24, no. 3-4, pp. 363-371, 1973.

[4] D. S. Lubinsky and T. Z. Mthembu, "Orthogonal expansions and the error of weighted polynomial approximation for Erdös weights," Numerical Functional Analysis and Optimization, vol. 13, no. 3-4, pp. 327-347, 1992.

[5] Á. Horváth, "Characterization of Fourier series with $(\mathrm{C}, 1)$ means," Rendiconti del Circolo Matematico di Palermo Serie II. Supplemento, vol. 68, pp. 491-511, 2002.

[6] D. S. Lubinsky and D. Mache, " $(C, 1)$ means of orthonormal expansions for exponential weights," Journal of Approximation Theory, vol. 103, no. 1, pp. 151-182, 2000.

[7] D. S. Lubinsky and H. P. Mashele, " $L_{p}$ boundedness of $(C, 1)$ means of orthogonal expansions for general exponential weights," Journal of Computational and Applied Mathematics, vol. 145, no. 2, pp. 387-405, 2002.

[8] H. Jung and R. Sakai, "Specific examples of exponential weights," Communications of the Korean Mathematical Society, vol. 24, no. 2, pp. 303-319, 2009.

[9] E. Levin and D. S. Lubinsky, Orthogonal Polynomials for Exponential Weights, Springer, New York, NY, USA, 2001.

[10] R. Sakai and N. Suzuki, "Mollification of exponential weights and its application to the Markov-Bernstein inequality," Pioneer Journal of Mathematic, vol. 7, no. 1, pp. 83-101, 2013.

[11] R. Sakai and N. Suzuki, "Favard-type inequalities for exponential weights," Pioneer Journal of Mathematics and Mathematical Sciences, vol. 3, no. 1, pp. 1-16, 2011. 


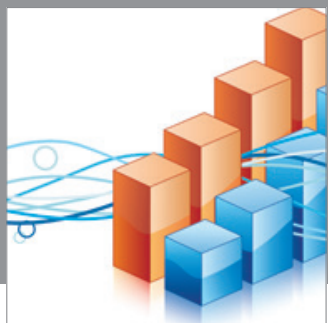

Advances in

Operations Research

mansans

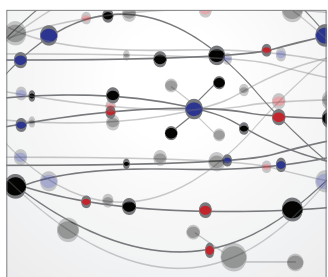

The Scientific World Journal
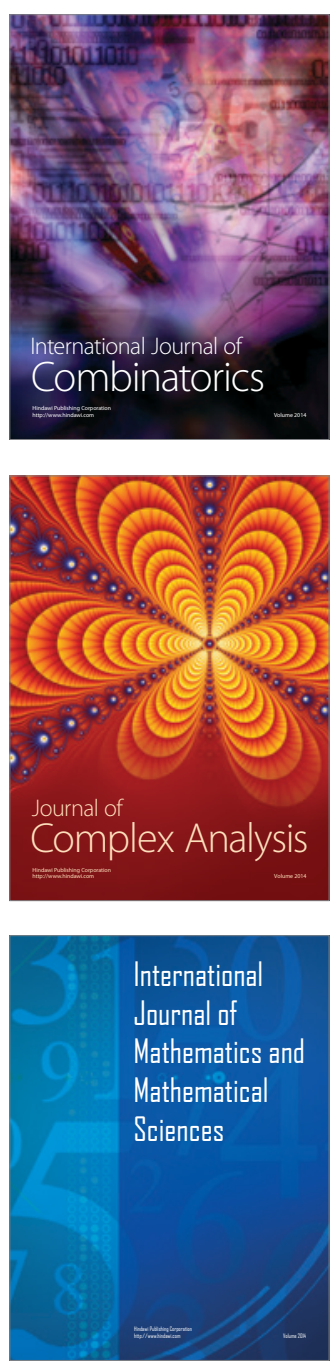
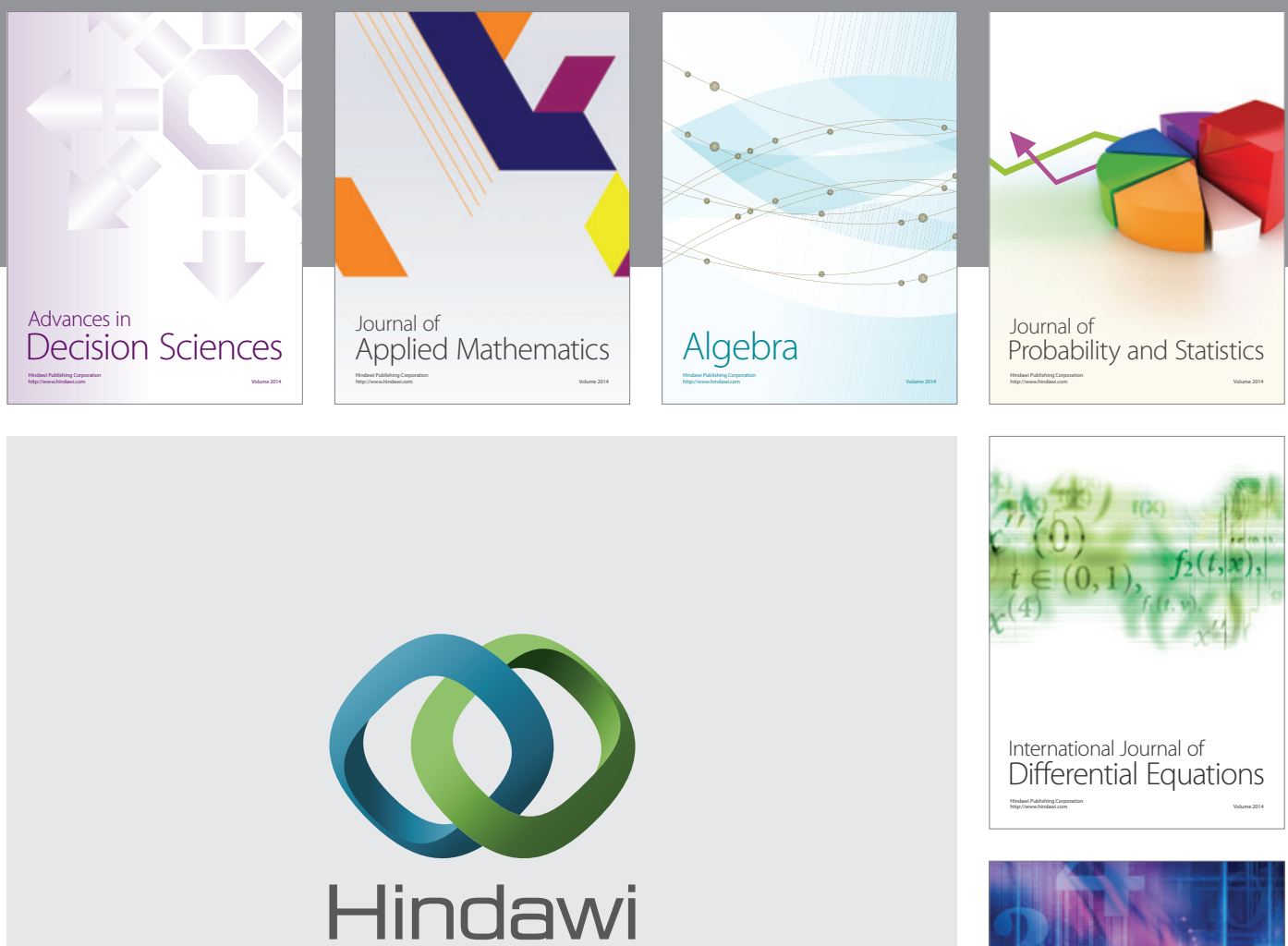

Submit your manuscripts at http://www.hindawi.com
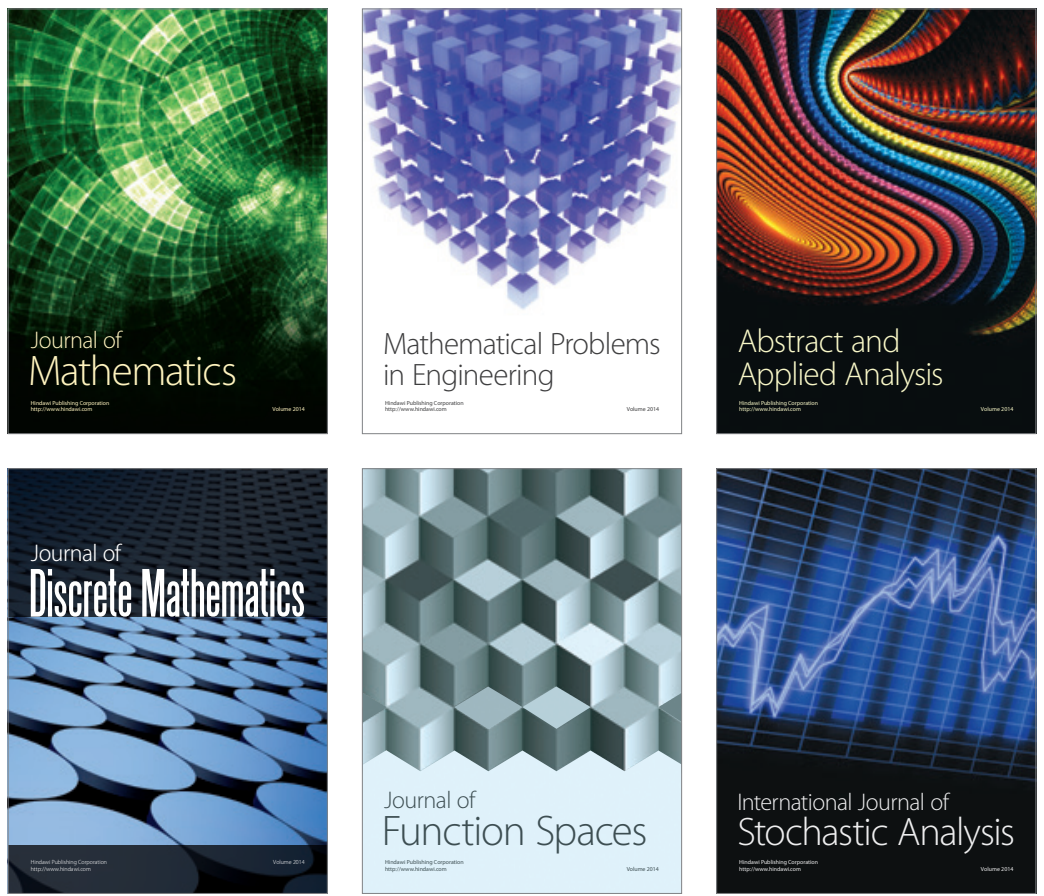

Journal of

Function Spaces

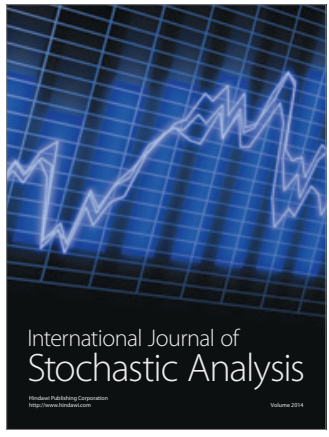

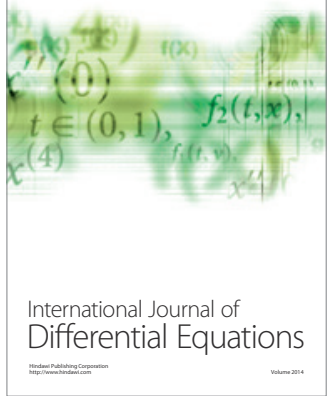
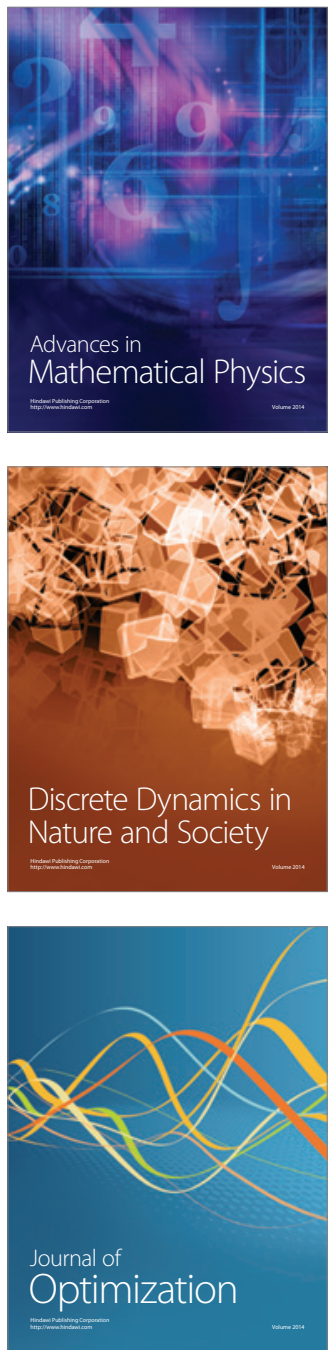